\title{
Optical coherence tomography reveals the mechanisms of balloon pulmonary angioplasty in inoperable chronic thromboembolic pulmonary hypertension
}

\author{
Aleksander Araszkiewicz, Stanisław Jankiewicz, Magdalena Janus, \\ Magdalena Łanocha, Tatiana Mularek-Kubzdela, Maciej Lesiak \\ $1^{\text {st }}$ Department of Cardiology, University of Medical Sciences, Poznan, Poland
}

A 76-year-old female patient with chronic thrombo-embolic pulmonary hypertension (CTEPH) as a result of previous pulmonary embolism was admitted for balloon pulmonary angioplasty. Right heart catheterization showed mean pulmonary artery pressure of $61 \mathrm{~mm} \mathrm{Hg}$. Selective pulmonary angiography revealed subtotal occlusion of the A8 segmental branch of the left pulmonary

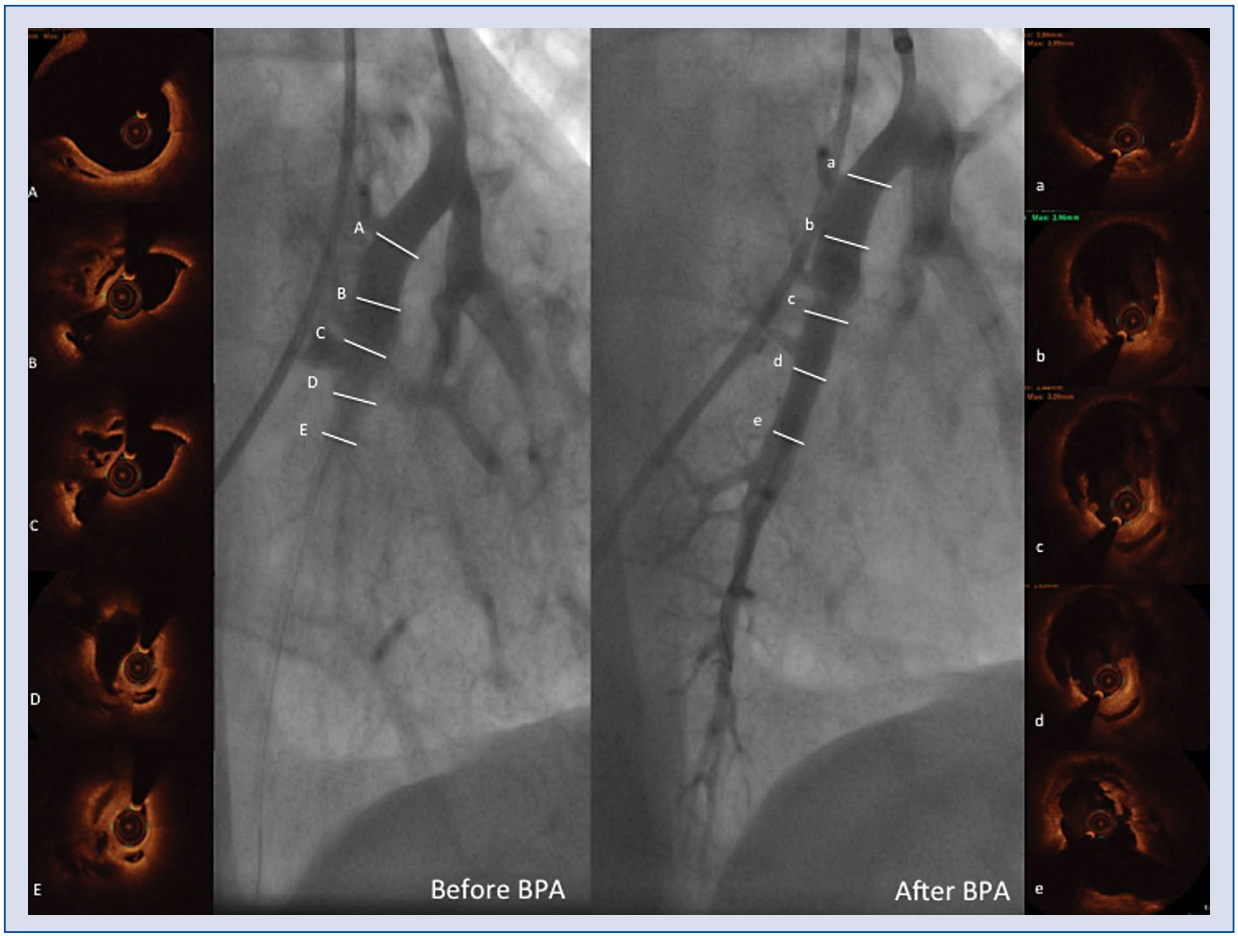

Figure 1. Angiography and optical coherence tomography (OCT) cross-sections of the eighth segmental branch of the left pulmonary artery, before and after balloon pulmonary angioplasty (BPA). A 6-French right Judkins guiding catheter was introduced to the ostium of the artery and 0.0014 " coronary guidewire was placed distally in the vessel. Then optical coherence tomography (OCT) catheter (DragonFly, St. Jude Medical, USA) was advanced. lodinated contrast was infused at a flow rate of $5 \mathrm{~mL} / \mathrm{s}$ over $4 \mathrm{~s}$ at 400 psi of pressure and OCT images were acquired. Subsequently the vessel was accurately measured in several locations and the proper size of the balloon was selected. OCT images revealed the potential mechanisms of BPA: expansion of internal lumen of the artery was achieved by breaking the meshwork lesions inside the artery lumen and by compression of the boundary white thrombus.

Address for correspondence: Aleksander Araszkiewicz, MD, PhD, $1^{\text {st }}$ Department of Cardiology, Poznan University of Medical Sciences, ul. Długa 1/2, 61-848 Poznań, Poland, tel: +48 608574375, fax: +48 618549223, e-mail: aaraszkiewicz@interia.pl 
artery. Optical coherence tomography (OCT) of the target vessel was performed to properly size the vessel and reduce the risk of post-reperfusion edema. OCT revealed extensive changes in the proximal- and mid-part of the target artery ("colander lesions" or meshwork) and subocclusion in the mid-distal part of the vessel. Five inflations of $2.5 \times 20 \mathrm{~mm}$ followed by three inflations of $4.0 \times$ $\times 15 \mathrm{~mm}$ semi-compliant balloon with the pressure of 4-10 atmospheres were performed with good angiographic and hemodynamic effect. OCT after the procedure showed lumen expansion, breaking the meshwork lesions inside the artery and a compression of the white thrombus. There were no complications in the peri- and post-procedural period.

Balloon pulmonary angioplasty is an emerging option for inoperable patients with CTEPH or with persistent pulmonary hypertension after pulmonary endarterectomy. OCT is a useful tool in determining of the location, extent and character of the pulmonary artery lesions and helps in selecting the appropriate balloon size and length. It could also decrease the risk of post-reperfusion edema. OCT could additionally be useful in determining the mechanism of balloon pulmonary angioplasty.

Conflict of interest: None declared 